\title{
Alteration of primary cilia in COPD
}

\author{
To the Editor:
}

Chronic obstructive pulmonary disease (COPD) is a major economic and social concern worldwide because of its impact on mortality and morbidity [1]. COPD is characterised by airway epithelium remodelling, a hallmark of dysregulated airway epithelium plasticity [2]. There are currently no available therapeutics to restore the integrity and functionality of the epithelium. Therefore, novel sources of investigation are becoming crucial to understand the alterations at the root of COPD initiation. Non-motile primary cilia are solitary sensor organelles playing a critical role in cell cycle control, proliferation, polarity and differentiation, particularly of ciliated cells possessing motile cilia [3, 4]. Primary cilia are assembled on different types of human cells depending on their state and activities in response to cellular quiescence where they relay extracellular signals and retract upon cell cycle re-entry [5]. Alterations of primary cilium structure and function are responsible for ciliopathies [6,7]. Primary cilia may be crucial in determining outcomes during airway epithelial cell differentiation thus we hypothesised that primary cilia are present in adult epithelial cells and may play a key role in airway plasticity. First, we investigated the presence and localisation of primary cilia in the bronchial epithelium. Secondly, we analysed the relationships between primary cilia and clinical, functional and histological characteristics of non-COPD and COPD patients.

Patients scheduled for lung resection for cancer (University Hospital of Reims, Reims, France) were prospectively recruited following standards approved by the institutional review board (IRB Reims-CHU-20110612). Informed consent was obtained from all the patients. Clinical assessment and pulmonary function tests were performed. Emphysema quantification on the resected lobe was performed visually on thoracic computed tomography scan by two independent investigators as previously described $[8,9]$. Formalin-fixed paraffin-embedded (FFPE) lung tissues distant from the tumour were stained with haematoxylin and eosin for bronchial epithelium analysis. Immunofluorescence was performed on FFPE lung tissues with the following primary antibodies: anti-Arl13b (1:200; ProteinTech, Rosemont, IL, USA); anti- $\gamma$-tubulin (1:200; Sigma, St. Louis, MO, USA); anti-acetylated- $\alpha$-tubulin (1:1000; Sigma), anti-GT335 (1:500; AdipoGen, Liestal, Switzerland), and anti-p63 (1:100; R\&D systems, Minneapolis, MN, USA). Images were taken by Confocal Zeiss LSM710 microscope (Oberkochen, Germany, objective 63x/1.40 oil DIC (differential interference contrast)). Primary cilia were analysed on 10 random fields per stained slide. Differences between groups were determined using the t-test or Fisher exact test and associations between variables were analysed using the Spearman rank correlation test. A p-value $<0.05$ was considered significant.

36 patients were included: 19 COPD patients (Global Initiative for Chronic Obstructive Lung Disease (GOLD) 1: $n=5$; GOLD 2: $n=12$; GOLD 3-4: $n=2$; age (median (interquartile range (IQR)) 66 years (59-73); forced expiratory volume in 1 s (FEV1, median (IQR): 71\% (62-81)), and 17 non-COPD patients (age 69 years (66-76); FEV1: 89\% (83-102)). Computed tomography emphysema score for the resected lobe was significantly higher in COPD group compared with the non-COPD group (0 (0-0) versus $1(0-2) ; \mathrm{p}<0.0001)$.

Cilia were identified and localised on epithelia with a specific staining for the cilia axoneme and membrane (GTPase Arl13b) and the basal body/centrosome ( $\gamma$-tubulin) (figure 1a). Motile cilia were readily identified at the surface of epithelial cells pointing towards the lumen, while solitary cilia (primary cilia) were found on non-differentiated cells of the pseudostratified epithelium as seen during mouse lung development [10]. We confirmed the identification of primary cilia with two additional and well-characterised markers of the axoneme (acetylated $\alpha$-tubulin and GT335) (figure 1a and data not shown) [11].

@ERSpublications

Non-differentiated airway epithelial cells display primary cilia and their number is associated with COPD features http://ow.ly/wiGH30jDbeG

Cite this article as: Perotin J-M, Coraux C, Lagonotte E, et al. Alteration of primary cilia in COPD. Eur Respir J 2018; 52: 1800122 [https://doi.org/10.1183/13993003.00122-2018]. 
a)
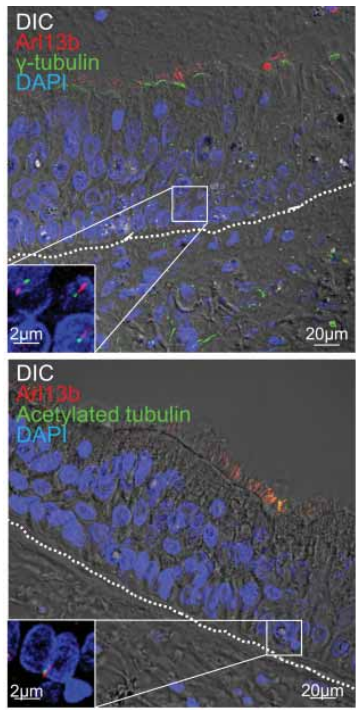

b)
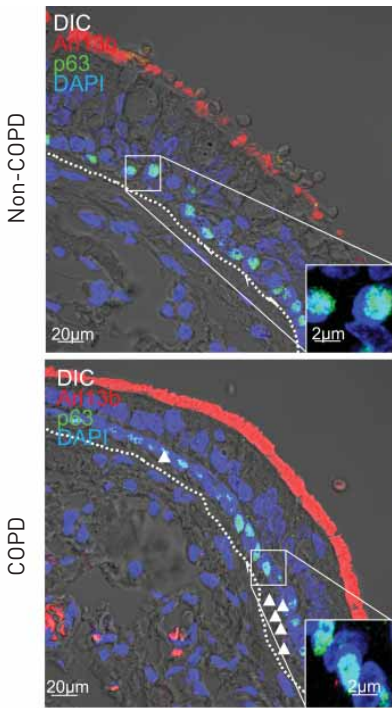
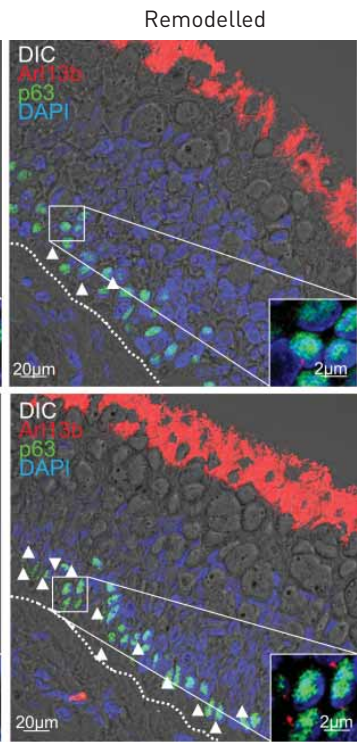
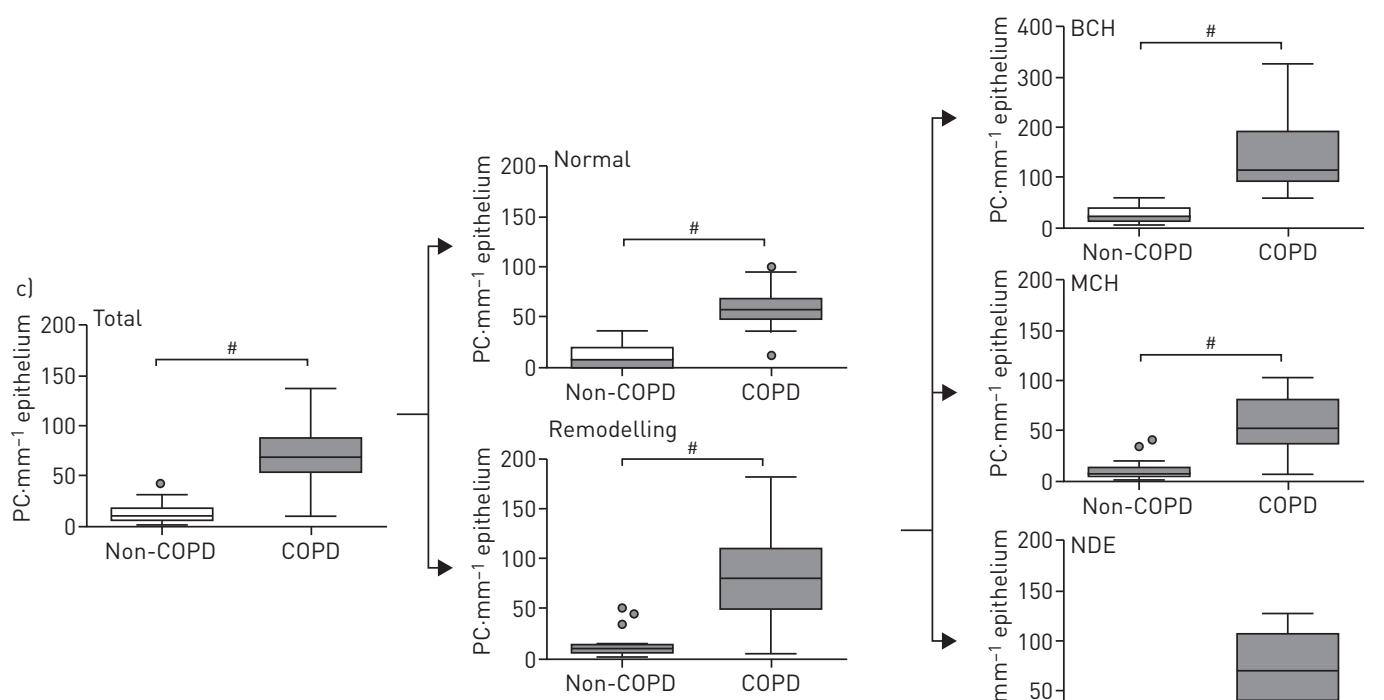

E $200 \mathrm{MCH}$
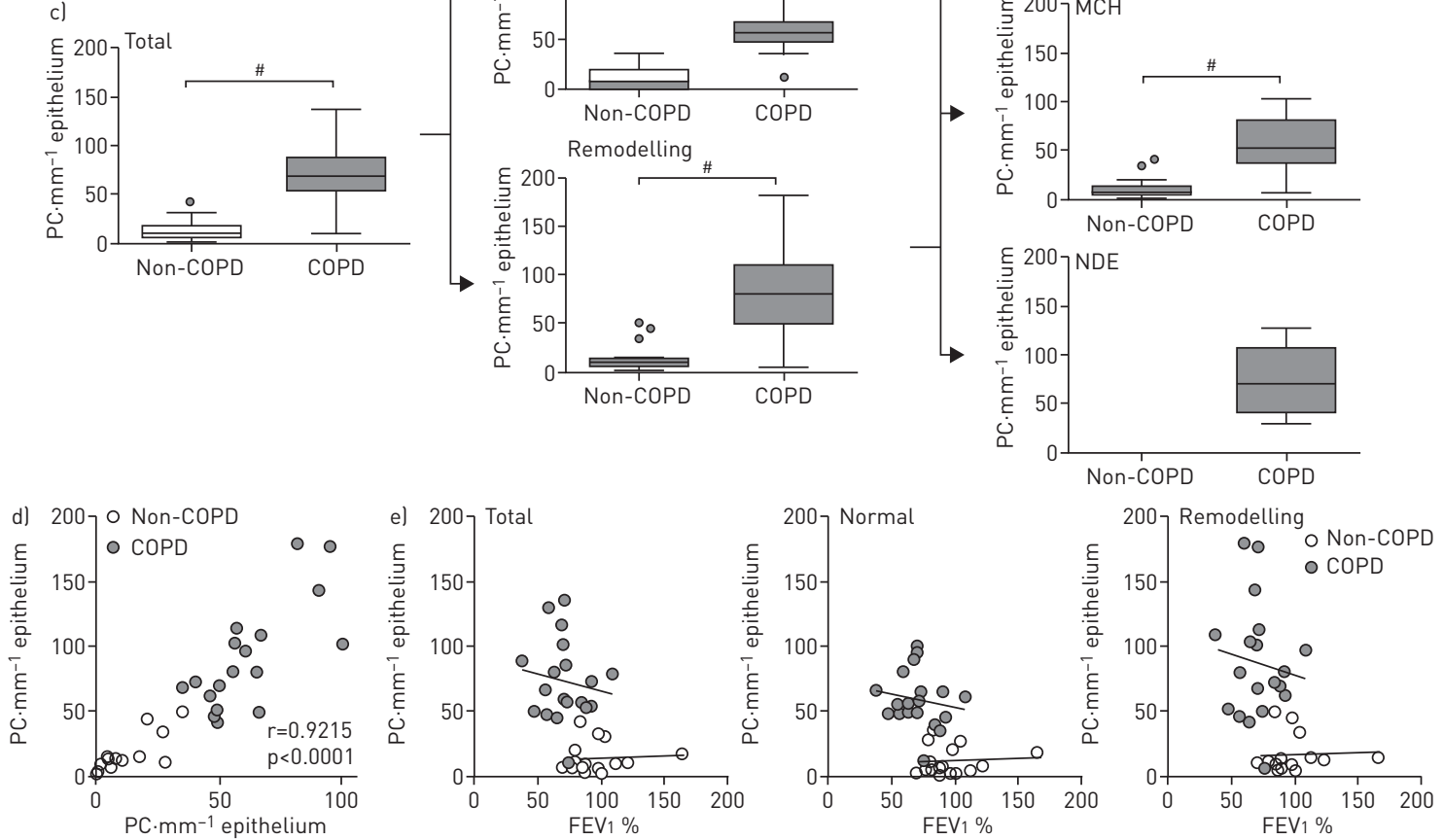

FIGURE 1 Primary cilia are present in adult bronchial epithelia and altered in chronic obstructive pulmonary disease (COPD) patients. a) Examples of maximum intensity z-stack projection showing the presence of primary cilium on confocal acquisition of the bronchial epithelia for two smoker

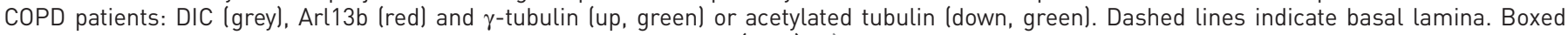
areas are shown as magnifications. Nuclei are stained with DAPI (blue). b) Representative maximum intensity z-stack projections comparing primary cilium on non-remodelled and remodelled areas for non-COPD and COPD patients on confocal acquisition for DIC (grey), Arl13b (red) and p63 (green). Arrowheads show primary cilium localisation. Dashed lines indicate basal lamina. Boxed areas are shown as magnifications. Nuclei are stained with DAPI (blue). c) Box and whiskers plot showing median with interquartile range of the numbers of primary cilia (PC) per epithelium types in non-COPD patients and COPD patients on the epithelium as a whole (total), normal epithelium (normal), remodelled areas (remodelling), basal cell hyperplasia (BCH), mucous cell hyperplasia (MCH) and non-differentiated epithelium (NDE). d) Graph depicting the repartition of non-COPD and COPD patients according to the number of primary cilia per mm of normal ( $\mathrm{x}$ axis) and remodelled (y axis) epithelium; e) Graphs depicting the repartition of non-COPD and COPD patients according to forced expiratory volume in $1 \mathrm{~s}$ (FEV 1 ) \% ( $\mathrm{x}$ axis) and the number of primary cilia per $\mathrm{mm}$ of epithelium (y axis) as a whole (left graph), normal (middle graph) or remodelled (right graph) areas. Lines indicate linear regressions. ${ }^{\#}$ : $p<0.0001$. 
Since it has been suggested that primary cilia are absent from adult lung tissues [10] while they are present on other human cells in resting phase [12], we considered to distinguish between histologically "normal" epithelia and "remodelled" epithelia [13]. A normal epithelium was defined as a pseudostratified epithelium: a) presenting the three main cell types (basal, ciliated and goblet cells); b) lacking hyperplasia or metaplasia; and c) showing at least $50 \%$ of ciliated cells at the surface.

We analysed bronchial epithelium in non-COPD and COPD groups (figure 1b). Taking the epithelium as a whole, COPD patients presented a tremendous increase in primary cilium numbers (median (IQR)) compared to non-COPD patients (68.1 (52.2-88.2) versus 9.5 (6.1-18.3); p<0.0001) (figure 1c). Interestingly, the normal epithelium of COPD patients showed an increase in primary cilium numbers compared with non-COPD patients (56.5 (48.2-67.1) versus $6.2(2.9-19.5) ; \mathrm{p}<0.0001)$ and this increase was more pronounced in remodelled areas (80.7 (51.1-109.4) versus 10.7 (7.9-14.8); $\mathrm{p}<0.0001)$. It was particularly striking in basal cell hyperplasia (116.9 (91.2-188.2) primary cilia per $\mathrm{mm}$ of epithelium in COPD patients versus 15.5 (8.9-32.5) in non-COPD patients; $\mathrm{p}<0.0001)$. Except for one subject, a number of 40 primary cilia per $\mathrm{mm}$ in normal epithelia and 50 primary cilia per $\mathrm{mm}$ in remodelling epithelia were identified as cut-off between COPD and non-COPD status $(\mathrm{r}=0.9215, \mathrm{p}<0.0001)$ (figure $1 \mathrm{~d})$. Interestingly, a significant increase of primary cilia was associated with smoking status $(p=0.01)$ but not with smoking history $(p=0.08)$, respiratory symptoms including dyspnoea score $(p=0.0003)$ and chronic bronchitis $(\mathrm{p}=0.04)$, the severity of airway obstruction ( $F E V 1: p=0.002$ and FEV1/forced vital capacity: $\mathrm{p}=8.7 \times 10^{-7}$ ) and the presence $(\mathrm{p}=0.008)$ and the severity of emphysema $(\mathrm{p}=0.0009)$. Each of these clinical or functional characteristics of patients coupled with the analysis of primary cilia set apart patients with $\mathrm{COPD}$, e.g. $\mathrm{FEV}_{1}$ (\% pred; figure 1e, the differences between elevations of linear regressions were extremely significant for each parameter in non-COPD versus COPD groups; $\mathrm{p}<0.0001$ ).

Localisation of primary cilia in the lung is consistent with their known functions including acquisition of polarity, migration, differentiation and cell cycle control [14]. Primary cilia were rare in epithelia from non-COPD patients suggesting that either primary cilia did not stabilise long enough to be observed or only a few cells were quiescent. The increase of primary cilia among COPD patients may pave the way to a novel understanding of cell plasticity in the context of this disease. 1) If primary cilia are cell cycle progression indicators, are the basal cells no longer able to divide to renew the epithelium? 2) Is the onset of COPD responsible for the apparition of primary cilium or vice versa? 3) Can an increase in primary cilia be considered as a marker of abnormal and dysfunctional bronchial epithelium that would be involved in the development of airway obstruction and/or emphysema, especially regarding to airway regeneration anomalies?

Interestingly, primary cilium numbers were altered between a normal and a remodelled area indicating that primary cilia may appear during the renewal or the repair of the epithelium [10]. This is also consistent with the known role of primary cilia in cell migration and tissue homeostasis [15]. Abnormalities of bronchial epithelial wound closure have been shown in severe COPD and were associated with the severity of airway obstruction and emphysema [8]. Likewise, anomalies of primary cilia could be involved in this phenomenon.

Some limitations must be pointed out in our study. Despite the novelty of the findings, these analyses are exploratory and conducted in a monocentric study including a relatively low number of patients. Thus, sample selection and cohort size represent biases, as well as the lack of "normal controls" which cannot be circumvent in studies conducted from lung resection tissues. Moreover, further investigations are clearly needed to precise the underlying mechanisms of primary cilium alteration.

In conclusion, we have shown an increase of primary cilia on bronchial epithelia associated with clinical, morphological, functional and histological parameters defining COPD. As the presence of primary cilium correlates with clinical features representative of COPD, understanding the dysregulation of primary cilium expression and function would provide additional clues in this complex pathology where the role of the epithelium appears increasingly important. To our knowledge, it is the first observation of non-motile primary cilia in human adult airway epithelia. Whether an accumulation of primary ciliated cells is a disease-driving process or a consequence of epithelium alterations will require further investigations.

Jeanne-Marie Perotin ${ }^{1,2}$, Christelle Coraux ${ }^{1}$, Eymeric Lagonotte ${ }^{1,3}$, Philippe Birembaut $^{1,3,4}$, Gonzague Delepine ${ }^{1,5}$, Myriam Polette ${ }^{1,3,4}$, Gaëtan Deslée ${ }^{1,2,3}$ and Valérian Dormoy $\oplus^{1,3}$

${ }^{1}$ Inserm U1250, University Hospital of Reims, Reims, France. ${ }^{2}$ Dept of Respiratory Diseases, University Hospital of Reims, Reims, France. ${ }^{3}$ University of Reims Champagne-Ardenne (URCA), SFR Cap-Santé, Reims, France. ${ }^{4}$ Dept of Biopathology, University Hospital of Reims, Reims, France. ${ }^{5}$ Dept of Cardio-Thoracic Surgery, University Hospital of Reims, Reims, France.

Correspondence: Valérian Dormoy, Inserm UMR-S 1250, University of Reims Champagne-Ardenne, CHU Maison Blanche, 45 rue Cognacq-Jay, 51092 Reims, France. E-mail: valerian.dormoy@univ-reims.fr

Received: Jan 192018 | Accepted after revision: April 112018 
Author contributions: Study concept: V. Dormoy; study design: J-M. Perotin and V. Dormoy; data acquisition: J-M. Perotin, E. Lagonotte, G. Delepine, G. Deslée and V. Dormoy; analysis and data interpretation: J-M. Perotin, G. Deslée, M. Polette and V. Dormoy; revision of manuscript: C. Coraux, P. Birembaut, M. Polette and G. Deslée; manuscript writing: J-M. Perotin and V. Dormoy

Conflict of interest: None declared.

Support statement: This work was supported by Région Champagne-Ardenne and the French National Institute of Health and Medical Research (Inserm). Funding information for this article has been deposited with the Crossref Funder Registry.

\section{References}

Rabe KF, Watz H. Chronic obstructive pulmonary disease. Lancet 2017; 389: 1931-1940.

Sohal SS. Epithelial and endothelial cell plasticity in chronic obstructive pulmonary disease (COPD). Respir Investig 2017; 55: 104-113.

3 Fu J, Hagan IM, Glover DM. The centrosome and its duplication cycle. Cold Spring Harb Perspect Biol, 2015; 7: a 015800 .

4 Malicki JJ, Johnson CA. The cilium: cellular antenna and central processing unit. Trends Cell Biol 2017; 27: $126-140$.

5 Goto H, Inaba H, Inagaki M. Mechanisms of ciliogenesis suppression in dividing cells. Cell Mol Life Sci 2017; 74: 881-890.

6 Mitchison HM, Valente EM. Motile and non-motile cilia in human pathology: from function to phenotypes. J Pathol 2017; 241: 294-309.

$7 \quad$ Braun DA, Hildebrandt F. Ciliopathies. Cold Spring Harb Perspect Biol 2017; 9: a028191.

8 Perotin JM, Adam D, Vella-Boucaud J, et al. Delay of airway epithelial wound repair in COPD is associated with airflow obstruction severity. Respir Res 2014; 15: 151.

9 Washko GR, Criner GJ, Mohsenifar Z, et al. Computed tomographic-based quantification of emphysema and correlation to pulmonary function and mechanics. COPD 2008; 5: 177-186.

10 Jain R, Pan J, Driscoll JA, et al. Temporal relationship between primary and motile ciliogenesis in airway epithelial cells. Am J Respir Cell Mol Biol 2010; 43: 731-739.

11 Hua K, Ferland RJ. Fixation methods can differentially affect ciliary protein immunolabeling. Cilia 2017; $6: 5$.

12 Fliegauf M, Benzing T, Omran H. When cilia go bad: cilia defects and ciliopathies. Nat Rev Mol Cell Biol 2007; 8 : 880-893.

13 Hirota N, Martin JG. Mechanisms of airway remodeling. Chest 2013; 144: 1026-1032.

14 Fry AM, Leaper MJ, Bayliss R. The primary cilium: guardian of organ development and homeostasis. Organogenesis 2014; 10: 62-68.

15 Veland IR, Lindbæk L, Christensen ST. Linking the primary cilium to cell migration in tissue repair and brain development. Bioscience 2014; 64: 1115-1125. 